\title{
Trial Expanded Access Status
}

National Cancer Institute

\section{Source}

National Cancer Institute. Trial Expanded Access Status. NCI Thesaurus. Code C127793.

The state or condition of an experimental drug or device as it pertains to its availability for use in expanded access studies. 